\title{
Modeling viral coevolution: HIV multi-clonal persistence and competition dynamics
}

\author{
F. Bagnoli ${ }^{\mathrm{a}, \mathrm{c}, *}$, P. Liò ${ }^{\mathrm{b}}$, L. Sguanci ${ }^{\mathrm{a}, \mathrm{c}}$ \\ ${ }^{a}$ Dipartimento di Energetica, Università di Firenze, Via S. Marta 3, 50139 Firenze, Italy \\ ${ }^{\mathrm{b}}$ Computer Laboratory, University of Cambridge, CB3 OFD Cambridge, UK \\ ${ }^{\mathrm{c}}$ INFN, sezione di Firenze and CSDC, Italy
}

Received 29 August 2005; received in revised form 4 October 2005

Available online 28 November 2005

\begin{abstract}
The coexistence of different viral strains (quasispecies) within the same host are nowadays observed for a growing number of viruses, most notably HIV, Marburg and Ebola, but the conditions for the formation and survival of new strains have not yet been understood. We present a model of HIV quasispecies competition, which describes the conditions of viral quasispecies coexistence under different immune system conditions. Our model incorporates both $\mathrm{T}$ and $\mathrm{B}$ cells responses, and we show that the role of B cells is important and additive to that of $\mathrm{T}$ cells. Simulations of coinfection (simultaneous infection) and superinfection (delayed secondary infection) scenarios in the early stages (days) and in the late stages of the infection (years) are in agreement with emerging molecular biology findings. The immune response induces a competition among similar phenotypes, leading to differentiation (quasispeciation), escape dynamics and complex oscillations of viral strain abundance. We found that the quasispecies dynamics after superinfection or coinfection has time scales of several months and becomes even slower when the immune system response is weak. Our model represents a general framework to study the speed and distribution of HIV quasispecies during disease progression, vaccination and therapy.
\end{abstract}

(C) 2005 Elsevier B.V. All rights reserved.

Keywords: HIV; Viral dynamics; Quasispecies; Coinfection; Superinfection

\section{Introduction}

The human immune system has the goal of providing a basic defense against pathogenic organisms [1,2]. The cells forming the immune system communicate via direct contact and through chemical signals. Using a very basic schematization, the immune system protective action can be divided into an innate, specific response, and an adaptive one. This latter component involves two major types of cells: lymphocytes ( $\mathrm{T}$ and $\mathrm{B}$ lymphocyte cells) and antigen presenting cells: monocytes, macrophages, B cells and dendritic cells. Lymphocytes have the ability to react to specific epitopes, which generally are portions of a protein. The idea is to recognize those shapes that constitute a fingerprint of the presence of a foreign agent (antigen), but not to

*Corresponding author. Dipartimento di Energetica, Università di Firenze, Via S. Marta 3, 50139 Firenze, Italy.

E-mail addresses: franco.bagnoli@unifi.it (F. Bagnoli), pietro.lio@cl.cam.ac.uk (P. Liò), luca.sguanci@unifi.it (L. Sguanci). 
react to self repertoire. Their response depends on the T cell receptor (TCR) of the T cell, and immunoglobulins (Ig) of the B cells. It is the great variety of cells carrying TCRs or secerning Igs that allows the recognition of all possible antigens.

Let us present a simplified description of the adaptive response, see Ref. [2] for a physicists-oriented introduction to immune system modeling. The task of protecting the body implies the detection and removal of free antigens, and the recognition and suppression of infected (or cancer) cells. These deviating self cells are identified by a sophisticated automatic mechanism, common to all cells, that presents on their surface specific receptors along with epitopes. Both viral infection or cancer proliferation implies the activation of novel genes, and thus the production of foreign proteins that can thus be detected. However, due to the danger (and cost) of an inflammation, the activation of the immune adaptive response is subject to a double key protection, implemented by $\mathrm{T}$ helper cells.

$\mathrm{T}$ cells are divided into CD4+ (helper) and CD8 + (killer) types, according with specific receptors present on their surface. CD4 $+\mathrm{T}$ cells provide the regulatory mechanism that triggers the immune response by finding and presenting antigens and releasing stimulatory signals (cytokines). T cells are specific for a given set of epitopes; simplifying, we can say that they are specific for a given antigen. Once activated, CD8 $+\mathrm{T}$ cells search for and kill other cells presenting the specific antigen. Their action is triggered by CD4 $+\mathrm{T}$ cells recognizing that antigen. This specificity is not absolute: there is a certain variability of receptors allowing cross-recognition of similar epitopes.

The coverage of all possible shapes is implemented by a unique mechanism of genetic combinatorial sampling. The protection against self-recognition is due to the maturation phase. Immature T lymphocytes are generated in the bone marrow and then travel to the thymus where they proceed through a selection process. In the thymus those $\mathrm{CD} 4+$ and $\mathrm{CD} 8+\mathrm{T}$ cells that initiate any reaction against self shapes are selected out.

$\mathrm{B}$ cells are responsible of the removal of extracellular antigens. Antigen specific CD4 $+\mathrm{T}$ cells are required to induce proliferation of antigen specific B cells. The activated B cells then proliferate and differentiate into plasma cells. These produce soluble counterparts to the cell's receptors, called antibodies, which circulate in the blood and fluids. Antibodies bind to specific extracellular antigens and allow them to be removed by other means.

An antigen selects a "strain" of lymphocytes which replicates in response (clonal response). During this growth, the specificity of receptors to a given antigen may be increased by an hypermutation mechanism that slightly modifies the genes specifying the receptor shapes. A few cells of this strain (memory cells) will persist for a long time in the body, after viral eradication: should the specific antigen reappear, the response will be faster and stronger (vaccination).

Let us now describe the immune response in the framework of an HIV infection. HIV infects cells that contain the CD4 receptor on their cell membrane, through which the virus attaches and enters the cell. The CD4 molecules are found on T lymphocyte helper cells, monocytes and macrophages.

As HIV uses the CD4 molecule to establish the initial binding to a cell, these cells are the main target for HIV infection. Since CD4 + T cells are vital to the establishment of an immune response, their progressive loss opens the way for AIDS-related opportunistic infections [3].

The worldwide presence of several strains of the HIV virus and their often simultaneous presence within a patient, due to the increased frequency of multiple infections, are the new remarkable features of HIV pandemia. For example, HIV-1, presently, exists as three groups classified according to their degree of similarity. Group M stands for main, Group O for outlier and Group N for non-M, non-O. Group M is the predominant group and is divided into nine strains (subtypes or strains) that differ in the env gene [4,5]. Specific strains are predominant in different geographical regions and their high mutation rates often allow researchers to track the network of contacts. A growing number of strains within each subtype is nowadays reported.

The origin of different HIV strains is related to the quasispecies characteristics of HIV evolution [6]. Quasispecies are the combined result of mutations and recombination, originating variability, and selection, which filters out this variability. The main source of mutations is the reverse transcription phase [1], in which the viral RNA is converted into DNA by viral proteins that are not very accurate. Other sources of variability are the errors during the transcription phase, deletions and other genetic rearrangements during the integration of viral DNA into the host chromosomes, including recombination with other viral DNA or 
proviruses present in the cell. This mechanism thus increases the viral diversity in the body. However, not all these variants have the same capability of completing their life cycle and multiplying their clone number (i.e., their fitness).

First of all, the infection capacity of mutants may vary, and also their speed of replication [7]. In some cases a single mutation is sufficient to alter significatively the fitness [8], or this may be due to the accumulation of several mutations (smooth or Fujiama landscape [9]). Since the number of targets (the substrate) is limited, the strains that originate more clones in the unit of time tend to eliminate less fit mutants, which are subsequently regenerated by the mutation mechanism [10]. While mutations are an essential ingredient for exploring the genetic space in the search for the fitness maximum, they also lower the average fitness of the strain, which generally is formed by a cloud of mutants around the fitness maximum (quasispecies). For a given fitness landscape, there is a maximum tolerable mutation rate above which the quasispecies structure is lost (error catastrophe [6]).

The HIV quasispecies seem able to compete and their persistence affects AIDS progression [11,12]. For instance, some mutants may even be unable to colonize new cells, but they may survive by parasitizing more invasive clones (this is particularly observed when recombination of several strains is present) [13]. Finally, the immune response is specific, with a certain tolerance, to a given antigen, and may vary from antigen to antigen, due, for instance, to their similarities to self shapes. Thus, the presence of an antigen originates an immune response that suppresses also variants of the stimulating clone. This results in a form of competition among strains or clones, which prefer to stay away in the genetic space in order to escape the immune response and/or lower their intensity by dividing the productive capacity of the immune system into several strains (multi-infection). The effect of this competition may result in a continuously varying dominant quasispecies that tries to escape the immune response, or the splitting of the quasispecies into multiple strains (quasispeciation). During the first phases of HIV infection, when the immune response is vigorous, the first phenomenon is observed until presumably the virus finds a niche in the genetic space, not sufficiently covered by the immune system, originating AIDS [14,15]. The resistance to antiviral treatment may be ascribed to a similar mechanism. When the immune system is depressed, also slightly less adapted strains may survive, giving origin to multi-clonal infections often observed in terminal patients [16].

Secondary HIV infections with closely related strains may induce the response and clonal selection of a new subsets of CD4 $+\mathrm{T}$ cells that recognize specifically the new HIV strain, even under conditions of chronic infection by other strains. We can distinguish two scenarios: strain coinfection (simultaneous infection) and superinfection (delayed secondary infection) scenarios (see for instance Refs. [17-21]).

Our aim is to model viral strain coevolution and understand the importance of factors such as mutation rates, strength of immune response, cross-talks between $\mathrm{T}$ and $\mathrm{B}$ cells and competition among strains in coinfection and superinfection.

The fate of an epidemic does not depend only on the evolution inside a single host, but also on the transmissibility to other hosts. The social factors involved in emergence of viral strains are diverse and include global transportation, urban crowding and poverty, changing behavioral patterns, human population growth, etc. [22]. We shall not deal with these aspects in the present paper.

The article is organized in the following way: in the following sections we introduce a model that combines $\mathrm{T}$ and $\mathrm{B}$ cells immune response to HIV infection. Then we extend the model to include quasispecies and we test the model in the scenarios of coinfection and superinfection using parameters derived from biological literature. Finally, we discuss results and work in progress.

In what follows, we shall use the terms strain and quasispecies, according with the scale at which one is examining the system. In order to resolve the quasispecies details, one needs to use a (genotypic or phenotypic) scale sufficiently fine to allow differentiation inside a single quasispecies. On the contrary, if one is interested in the ecological relations among quasispecies, it is more efficient to use a scale in which a whole quasispecies is simply collapsed in a single value. In this latter case we use the term strain to refer to the whole quasispecies.

\section{Models}

Mathematical models have proven valuable in understanding the mechanisms of many of the observed features of HIV dynamics, such as the positive and negative regulation of $\mathrm{T}$ and $\mathrm{B}$ cell selection, the dynamics 
of production of the TCR repertoire in the thymus, the progression to and the latent phase of AIDS [15,23-29]. They have also been useful in forecasting the effects of multi-drug therapy.

While the majority of HIV dynamics models assume that each CD4 $+\mathrm{T}$ cell is infected by a single HIV strain, Dixit and Perelson [30] have shown that with the progression of HIV infection multiple infections become very likely.

Our goal was to investigate the conditions of the multi-strain persistence within a single patient and the effects of coinfection and superinfection. To set the scenarios of our model, it is noteworthy that, in general, the immune response can be seen as a prey/predator dynamics, in that the immune system is the predator of the virus (the prey). The HIV dynamics is more complex because the HIV is both the prey and the predator, i.e., it attacks CD4 $+\mathrm{T}$ cells and is attacked by cells coordinated by CD4 $+\mathrm{T}$ cells.

Fig. 1 illustrates the relationships among the different quantities of our model. This schematization is valid for the quasispecies case and for the undifferentiated one. In this latter case, the indication of strains (in parenthesis) should be neglected. The clonal amplification of naive T cells (of population $i$ ) depends on the ability of (ith class) $\mathrm{T}$ cell to recognize all the infected $\mathrm{T}$ cells (carrying an epitope from the $k$ th class of viruses). The rate of infection of naive T cells (of class $i$ ) depends on all the viruses (containing the epitope $k$ ). In the same way, the clonal expansion of infected $\mathrm{T}$ cells (of class $k$, meaning that they have been infected by a virus of class $k$ irrespective of the original T class) depends on the interaction of these viruses with all the T cells (of any class $i$ ).

\subsection{Combining $B$ and $T$ cell responses}

Let us start from a widely accepted theoretical model of HIV progression and immune response proposed by Perelson and colleagues in 1995 [15], recently extended to include CTL response and stochastic components [23]. There are several works that discuss the role of $B$ cells in the immune response [14]. Since the progression to AIDS has been found to correlate well with CD4 $+\mathrm{T}$ cells decrease, B cells are thought to play a minor role in the immune response to HIV. Note that B cells can act only as predator to the HIV, so their coupling with HIV dynamics is different from that of T cells. Our aim is to present a more general model framework of both

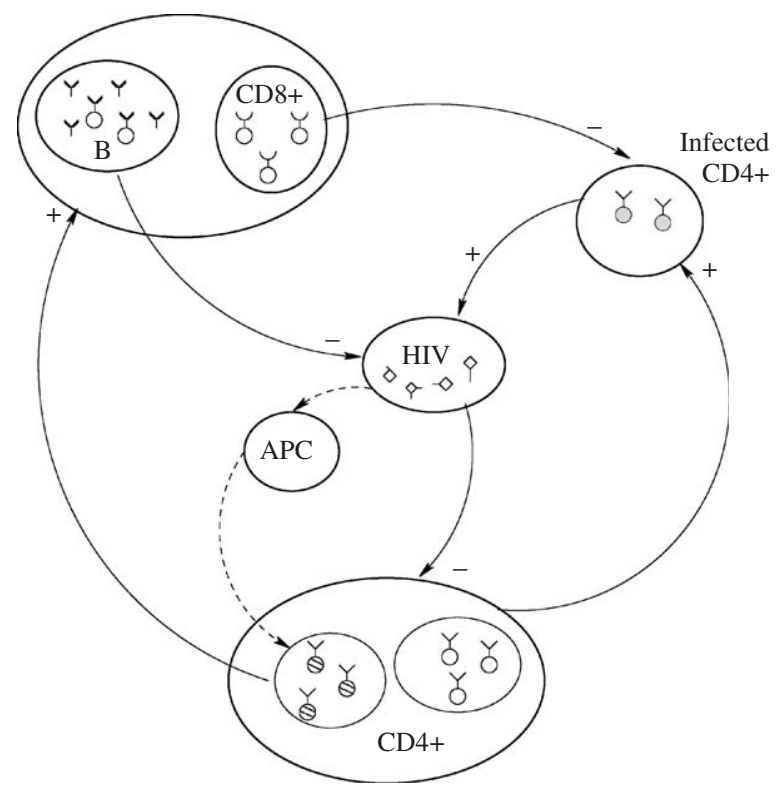

Fig. 1. Representation of the interactions between cells and/or cells and viruses. HIV strains infect CD4 + T cells that become infected and produce new viruses. At the same time HIV peptides are presented via APC cells to T helper cells that become activated. Activated CD4+ $\mathrm{T}$ cells trigger $\mathrm{B}$ and $\mathrm{CD} 8+$ cell reactions: the first release antibodies that bind to the antigen while the latter directly remove infected CD4+ T cells. "+" and "-" signs indicate cell/virus production or removal. 
$\mathrm{T}$ and $\mathrm{B}$ immune responses to HIV. We have first considered the following system of differential equations describing the dynamics of a single viral strain:

$$
\begin{aligned}
& \dot{T}=\left(\lambda+\gamma^{(T)} I T\right)(1-T / K)-\left(\delta_{T}+\beta V\right) T, \\
& \dot{I}=\beta V T-\left(\delta_{I}+\gamma^{(I)} T\right) I, \\
& \dot{V}=\pi I-\left(c+\gamma^{(V)} T\right) V .
\end{aligned}
$$

All quantities indicated by Greek letters and $c$ have unit days ${ }^{-1}$.

This model considers the T-helper $(\mathrm{CD} 4+)$ cells $(T)$, and HIV virus particles $(V)$; the $\mathrm{T}$ cells can become infected (I). With respect to Refs. [15,23], in Eq. (1), we describe how the number of naive T cells $(T)$ which have passed the thymus selection depends on rate of formation in the bone marrow $(\lambda)$, and on clonal amplification upon stimulation by infected cells, $I$ (term $I T)$. They decrease with a rate that is the sum of a natural clearance, $\left(\delta_{T} T\right)$, due to cell aging, and cell destruction upon virus infection, $(V T)$. The density of $T$ cells is limited by a saturating density/lymphonode capacity factor, $K$. Following Ref. [28] we have set $K=10^{12}$.

The second equation describes the rate of infection, described by $\beta$, of naive $\mathrm{T}$ cells upon the interaction with the virus (term $V T$ ). Infected $T$ cells are cleared out at a fixed rate, $\delta_{I}$, and due to the action of natural killer cells, CD8+ (term $T I)$.

The third equation describes the budding of viruses from infected cells, $\pi$. Virus particles are cleared out at rate $c$ (defective viruses) and after immunoglobulin binding and subsequent engulfments by the macrophages (term $T V$ ).

The $\gamma$ parameters have the same meaning of the constant of association in chemistry, or can be thought as a combination of both the probability of interaction and the interaction strengths between cells $\left(\gamma^{(T)}, \gamma^{(I)}\right)$ or between cells and viruses $\left(\gamma^{(V)}\right)$. Note that in the limit $\gamma \rightarrow 0$ and $K \rightarrow \infty$, we recover the pattern of the standard model [27].

The $\mathrm{B}$ cell response is modeled using the parameters corresponding to the $\mathrm{T}$ cells which activate them by receptor recognition. Here, we have assumed the immunoglobulin concentrations, which represent the $\mathrm{B}$ cell response, are linearly correlated to the concentration of activated B cells. These in turn are supposed linearly correlated to the concentration of the $\mathrm{CD} 4+\mathrm{T}$ cells. Exploratory analysis with different parameters suggested us that Eq. (3) allows to keep a minimum number of parameters without loss of important details.

Fig. 2 shows some typical behavior of this single-species model, showing the case of asymptotic coexistence of immune system and virus (chronical infection) and virus eradication. This latter scenario may be triggered both by increasing the T killer $\left(\gamma^{(I)}\right)$ or B $\left(\gamma^{(V)}\right)$ response. In no cases it is possible to observe the defeat of the immune system, since a decrease of the quantity of $\mathrm{T}$ cells affects also the rate of viral production. This reflects
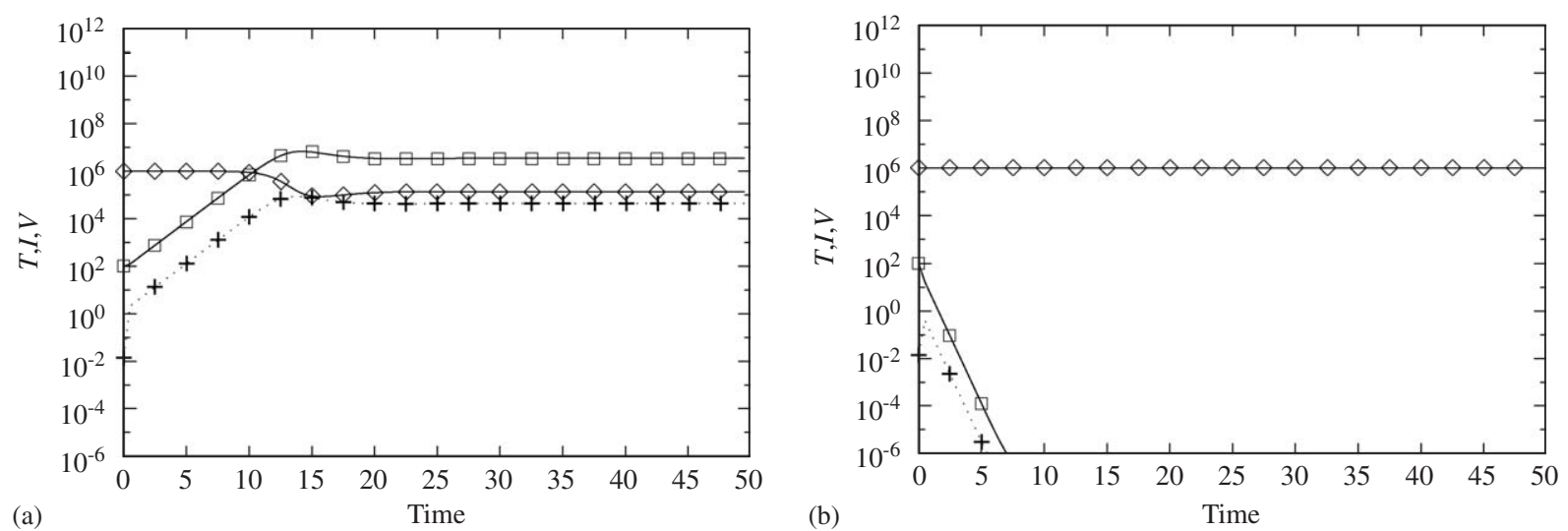

Fig. 2. Typical time evolution of the single-species model; diamonds represent uninfected $\mathrm{T}$ cells, squares represent viruses and plus signs the infected T cells. Plot (a) illustrates a scenario leading to a chronic infection $\left(\gamma^{(T)}=10^{-6}, \gamma^{(I)}=10^{-5}, \gamma^{(V)}=0\right)$. In the presence of a further B cell response (plot (b)), the infection is defeated by the immune system $\left(\gamma^{(T)}=10^{-6}, \gamma^{(I)}=10^{-5}\right.$ and $\left.\gamma^{(V)}=5 \times 10^{-6}\right)$. 
the observed fact that progression to AIDS and death is caused by opportunistic infections and not directly by the HIV virus. Model parameters are derived from medical literature (see also Ref. [23]).

\subsection{Modeling quasispecies competition and persistence}

Our actual model is obtained by extending Eqs. (1)-(3), to include the effects of quasispecies persistence.

For the sake of simplicity, we assume that each viral strain is characterized by just one epitope $i$. We make use of coupled differential equations, one for each viral quasispecies and $\mathrm{T}$ cell. Although this mean-field approach disregards the effect of fluctuations and genetic drift in quasispecies abundances, it is useful for understanding the coarse-grain features of the behavior of the interplay between HIV and the immune system.

The quasispecies model is described by the following set of equations:

$$
\begin{aligned}
& \dot{T}_{i}=\left(\lambda_{i}+\sum_{k} \gamma_{i k}^{(T)} I_{k} T_{i}\right)\left(1-\frac{1}{K} \sum_{i} T_{i}\right)-\left(\delta_{T}+\sum_{k} \beta_{k} V_{k}\right) T_{i}, \\
& \dot{I}_{k}=\left(\sum_{k^{\prime}} \mu_{k k^{\prime}} \beta_{k^{\prime}} V_{k^{\prime}}\right)\left(\sum_{i} T_{i}\right)-\left(\delta_{I}+\sum_{i} \gamma_{k i}^{(I)} T_{i}\right) I_{k}, \\
& \dot{V}_{k}=\pi I_{k}-\left(c+\sum_{i} \gamma_{k i}^{(V)} T_{i}\right) V_{k} .
\end{aligned}
$$

The model considers the following cell types: T-helper $(\mathrm{CD} 4+)$ cells responding to virus strain $i,\left(T_{i}\right)$; T cells (any strain) infected by virus strain $k,\left(I_{k}\right)$; abundance of viral strain $k,\left(V_{k}\right)$. This means that viral strain $k$ are identified by just one epitope, which is then displayed on the surface of the T cell of class $k$, and that a $\mathrm{T}$ cell of class $i$ can be activated at least by one CD4 + T cell carrying the epitope $k$, which is specific of the viral strain $k$. The indices $i(k)$ range from 1 to $N_{i}\left(N_{k}\right)$, and in the following we have used $N_{i}=N_{k}=N$.

Eq. (4) describes the generation of $T$ cells through two mechanisms: the bone-marrow source (and selection in the thymus) and the duplication of $T$ cell strains activated upon the recognition with an antigen carrying cell that may be even an infected one. In order to take into account the limits of the immune response, we have investigated both a logistic term and different types of saturating and density-dependent functions [23]. The logistic term models the global carrying capacity of immune system. In other works [23], a saturating function or density dependent function was used, aiming to describe how the growth and development of the immune response is limited by the timing of $\mathrm{T}$ cell division among other constraints such as cell-cell recognition, signal diffusion, and cell migration.

The death rate term represents both a natural death rate proportional to the population, and the infection rate of $\mathrm{T}$ cells by any viral strain. The infection probability, reflected by the term $\sum_{k} \beta_{k} V_{k} T_{i}$ and by the sum over $T_{i}$ in the $I$ cell birth rate, is the same irrespective of the $\mathrm{T}$ class. We assume that an infected $\mathrm{T}$ cell no more contributes to the immune response.

Eq. (5) describes the infection dynamics. The incorporation of two death rate parameters reflects the fact that the infected cells disappear due to cellular death and after the action of natural killer cells $(C D 8+)$. There are clear experimental evidences that CD4 + cells decrease during the late HIV infection stages and in the AIDS state. In terms of our models this mean that the parameter $\delta_{I}$ depends on the stage of the infection. We have preferred to model the early stage of infection, far from AIDS conditions and therefore we have set $\delta_{I}$ to a constant, medical literature referred, value.

The term $\mu_{k k^{\prime}}$ represents a mutation process that affects the phenotype. Mutations occur mainly during the reverse transcriptase processing of the viral genomes. The mutation parameter is essential for the formation of new quasispecies. RNA viruses have been reported to have substitution rates of the order of $1 \times 10^{-3}$ substitution per site per replication [31]. Mansky and Temin [32] determined an in vivo mutation rate of $3.5 \times 10^{-5}$. The majority of mutations does not change the amino acids. Moreover, most of amino acid substitutions are neutral or quasi-neutral, since they do not change remarkably the protein structure, and therefore the fitness of the species; effective differences in fitness and behavior evolve through several amino 
acid changes (see for instance Ref. [33]). Nevertheless, one single event of recombination can often alter the fitness in a substantial way. We take into consideration only those non-synonymous mutations that alter the phenotype (protein structure), and therefore we used a slightly smaller value for the mutation rate of the order of $10^{-5}$.

We have implemented the matrix $\mu_{k k^{\prime}}$ as a discrete Laplacian (diffusion in phenotypic space) controlled by parameter $\mu$ (without indices).

Eq. (6) describes the virus replicative dynamics in terms of a birth rate proportional to the virus "budding" numerosity. The viral death rate parameters depend on the rate of natural death and on the recognition of virus by $\mathrm{B}$ cells.

As in the single-specie model, B cells and T killer cells are only implicitly included in the model in order to reduce the dimensionality without losing too many details. We assume that these responses are fast enough to be at equilibrium and they are just proportional to the abundance of (cognate) $T$ helper cells.

While in the single-strain model, Eqs. (1)-(3), the three $\gamma$ parameters are scalar values, here $\gamma_{i k}^{(T)}, \gamma_{k i}^{(I)}$ and $\gamma_{k i}^{(V)}$ are matrices describing the interactions between viral strains and immune cells, i.e., who will interact with whom. The $\gamma$ parameters represent the interaction between cells and/or cells and viruses in terms of geometry and strength of the interaction. It expresses in a single matrix all the information about diffusion, epitope recognition and show some analogies with a constant-in-time fitness, as described in Ref. [34].

When the $\gamma$ matrices are diagonal, each $\mathrm{T}$ cell interacts only with one viral strain. The non-zero elements of the $i$ th row in the $\gamma$ matrices represent the strains of the virus recognized by the immune cells of class $i$ while the value represents the affinity maturation, i.e., the accuracy in recognition.

The $\pi$ coefficients (all equal), the $\gamma^{(T)}$ matrix and other parameters will contribute to the replicative success of the virus, i.e., its fitness. Many of the parameters are set equal among the different strains. Therefore, the $\gamma_{i i}^{(T)}$, which represents the efficiency of the $\mathrm{T}$ cell recognition of viral antigens, is the most important determinant of the viral fitness.

In order to control the shape of the $\gamma$ matrices with few parameters, we choose the following functional form (see Fig. 3):

$$
\gamma_{i j}=A\left\{\frac{1}{2}\left[1+\cos \left(\frac{2 \pi}{N}(i-j)\right)\right]\right\}^{\varepsilon_{A}}+B\left\{\frac{1}{2}\left[1+\cos \left(\frac{\pi}{N}(i+j)\right)\right]\right\}^{\varepsilon_{B}} .
$$

The parameters $A$ and $\varepsilon_{A}$ ( $B$ and $\varepsilon_{B}$ ) control the shape of the $\gamma$ matrices in the direction transverse (parallel) to the main diagonal. The exponents $\varepsilon_{A}$ and $\varepsilon_{B}$ control the smoothness of the variation along the corresponding direction. For $\varepsilon$ small the variation of the function is very small, while for $\varepsilon \rightarrow \infty$ the corresponding function

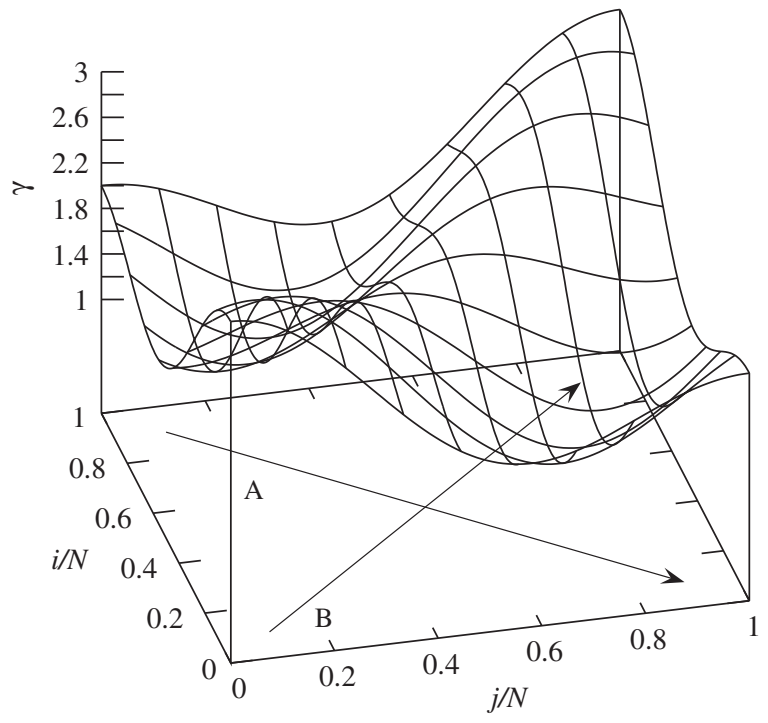

Fig. 3. Control parameters and shape of the $\gamma$-matrices. 
is $\delta$-shape. So, a diagonal $\gamma$ matrix is obtained by setting $B=0$ and $\varepsilon_{A}$ large. For instance, differences in the fitness of phenotypes may be obtained by setting $B$ and $\varepsilon_{B}$ different from zero in some of the $\gamma$-matrices, while the intra-species competition is triggered by setting $A$ different from zero and $\varepsilon_{A}$ small.

It is worth noting that nowadays, 2-photon experiments allow to measure recognition strength, movements and speed of the immune cells [35] that, in theory, can be used to derive estimates of the $\gamma$ matrices. We think that these data are still in embryonal phase, so we focus on the qualitatively features of the response.

We introduce the assumption of a linear strain space that represents the different phenotypes, see for instance Refs. [41,42] for similar assumptions. The strain space is ordered in terms of phenotype similarity. This assumption is justified if the phenotypes are determined by few viral protein functional determinants which are both independent and differ only in few DNA bases, i.e., few mutations can change one determinant into another. Since we consider a quasi-neutral model of evolution, e.g., sequential mutations change one amino acid into another similar, boundaries can be modeled as periodic. These two assumptions, linear space and periodic boundaries, certainly make the model more manageable.

We have used the same values of model parameters by re-scaling $T, I, V, \lambda_{i}$ and $K$ by the number $N$ of strains.

\section{Numerical results}

The evolution of $\mathrm{T}$ cell abundances in a scenario of quasispecies is shown in Fig. 4. Note that when the asymptotic state of our model is given by a fixed point, the asymptotic distribution is insensitive of the initial conditions, and the strains corresponding to higher fitness are more abundant. However, one should consider that this asymptotic state may be reached after such a long time that it may be outside any practical scenario of the progression of a disease. The role of mutations in the transitory regime is quite particular. First of all, mutations are necessary to populate strains outside the first inoculum, see also Fig. 6. Mutations affect also the average fitness and the width of the quasispecies and the Eigen's error threshold [36] is a sort of extremal consequence of this effect. However, in the presence of coupling among strains, due to competition or to global constraints (the $K$ parameter), the specific form of mutations does not play a fundamental role, see also Ref. [34].

\subsection{Phase diagrams of $\gamma$ matrices}

We focused our attention on the parameter $A$ of the three $\gamma$ matrices which represent the major features of the interaction, Fig. 5. The regions I and II in the three diagrams represent chronic infection (coexistence of
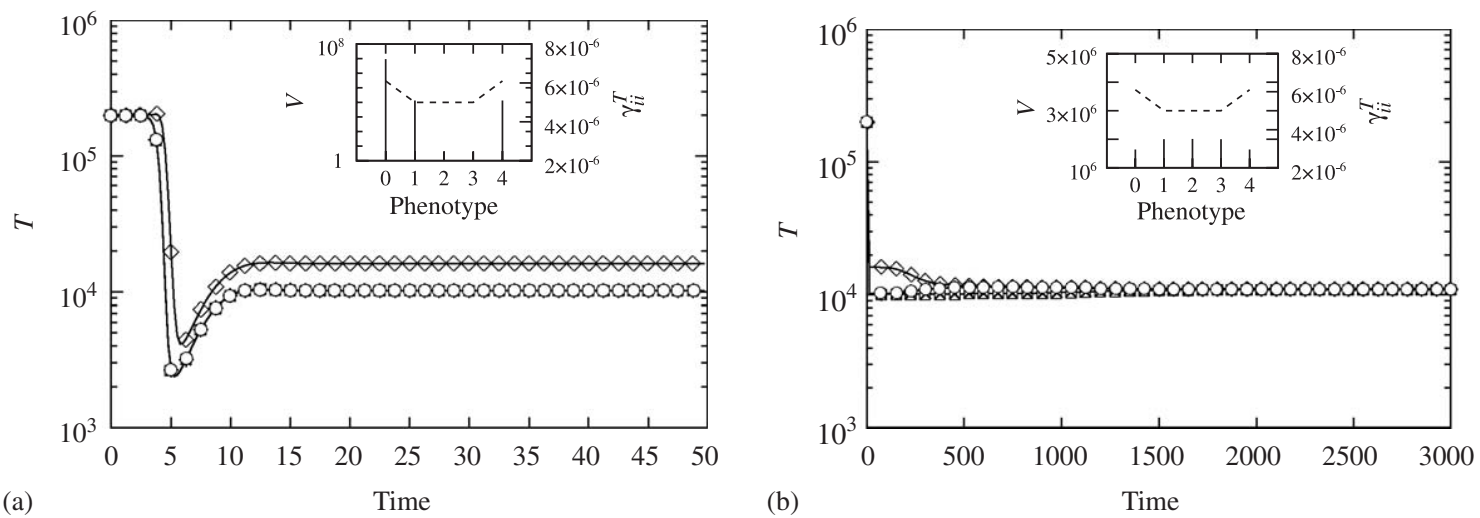

Fig. 4. Typical time evolution of the $\mathrm{T}$ cell abundance in the multi-species model for short-term (a) and long-term behavior (b). We set $\mu=10^{-5}, A=5 \times 10^{-6}, \varepsilon_{A}=10, B^{(T)}=3 \times 10^{-6}, \varepsilon_{B}^{(T)}=10$ with $N=5$. In the inset, the $y$-axis on the left reports the asymptotic abundance of virus strains, the $y$-axis on the right shows the interaction strength (dashed line) between $\mathrm{T}$ cells and virus phenotypes (x-axis). 

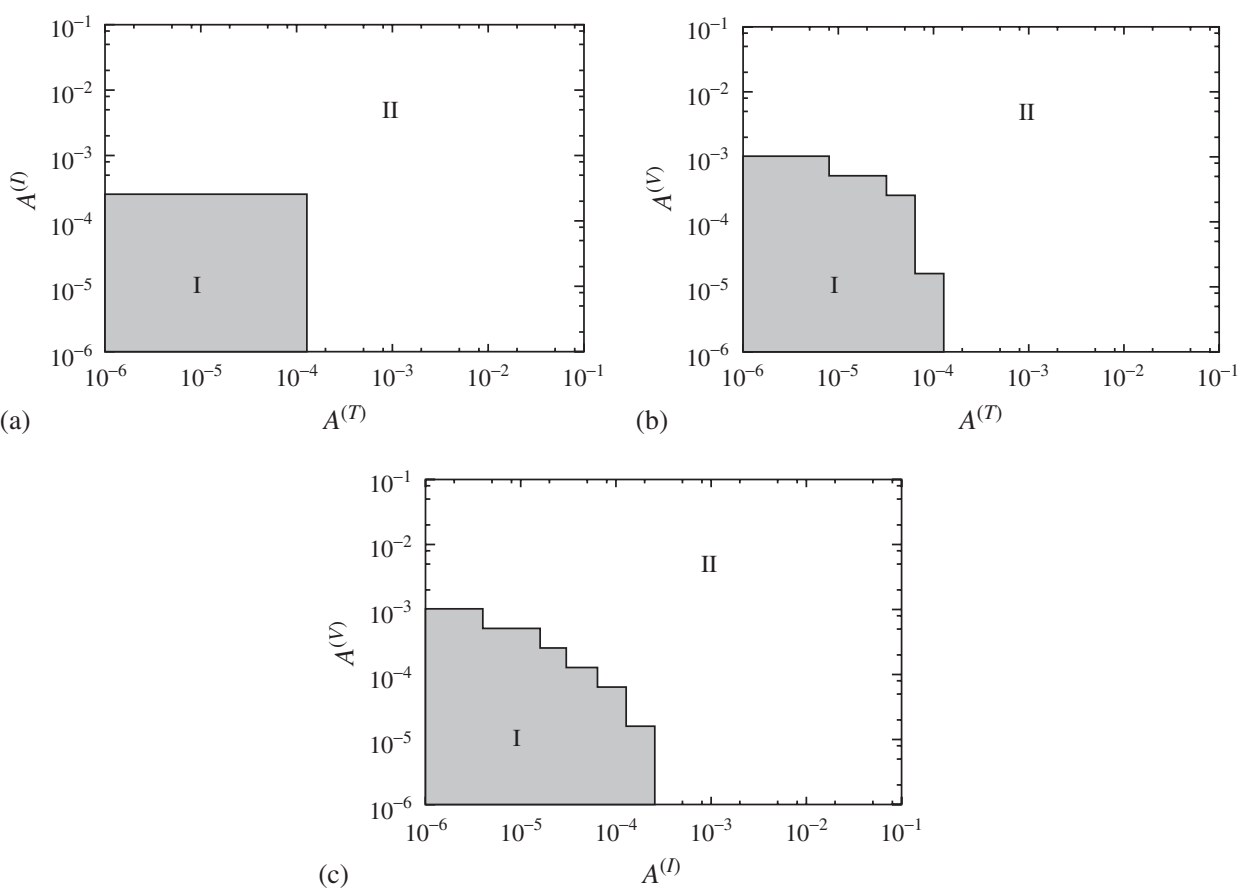

Fig. 5. Phase space analysis of $A$ s parameters of the $\gamma$ matrices. In (a) we fix $A^{(V)}=10^{-6}$ and we show $A^{(I)}$ vs. $A^{(T)}$. In (b) we fix $A^{(I)}=10^{-6}$ and we show $A^{(V)}$ vs. $A^{(T)}$. In (c) we fix $A^{(T)}=10^{-6}$ and we show $A^{(V)}$ vs. $A^{(I)}$. The other parameters are: number of quasispecies $N=25, \varepsilon_{A}=10^{3}, B=0$ and $\mu=0$. Region I corresponds to the coexistence of immune system and viruses (chronic infection) while region II corresponds to the defeat of viral infection.

immune system and viral infection) and complete recovery (virus defeat) from the disease, respectively. For the sake of clarity instead of $3 \mathrm{D}$ diagrams, we choose $2 \mathrm{D}$ projections, after setting the other dimension to a biologically meaningful value.

The effect of B cells is shown in Fig. 5a : for a very low contribution of B cells to the immune response, there is a clear threshold effect on both $\gamma^{(T)}$ and $\gamma^{(I)}$. By considering a weak action of T killer cells, the threshold effect for $\gamma^{(T)}$ and $\gamma^{(V)}$ (B cells effect) is somewhat decreased but still present, Fig. 5b. Instead, Fig. 5c shows that the combined effects of $\mathrm{B}$ and $\mathrm{T}$ cells are almost additive, despite the parameter $K$ that limits the total population of T cells. For example, let us look at Fig. 5c. If we draw a line at $A^{(I)}=2.56 \times 10^{-4}$, which is an extreme value for coexistence in Fig. 5a, we can notice that both the two phases are present, in particular there are values of $A^{(V)}$ leading to the defeat of the infection.

\subsection{Coinfection dynamics}

The effect of competitive evolution observed after a single inoculum of two different HIV strains at time $t=0$ is shown in Fig. 6. We have considered two different scenarios. The first one (Fig. 6a, b) represents the time evolution of $\mathrm{T}$ cells and viruses when the epitopes of two different strains are subjected to the same interaction strength. The second scenario (Fig. 6c, d) considers differences in the recognition ability of viral antigens by $\mathrm{T}$ cells. For example, variants of the CCR5 receptors may induce partial resistance against HIV [37]. De Boer and Perelson have shown [25] that the phase space of the antigen-T cell recognition is not homogeneous but it is patched with areas of strong immune response and areas of lack of immune response. This difference in the strains targeted by the $\mathrm{T}$ cells generates changes in the fitness of the virus that turn into differences in their abundances. The immune system response is almost the same in the two cases, and very similar to that obtained without considering the phenotypic space, Fig. 2. The asymptotic distribution of viruses reflects the behavior of the fitness is induced by the stimulation of the immune response, $\gamma_{i i}^{(T)}$, as in Fig. 4. 

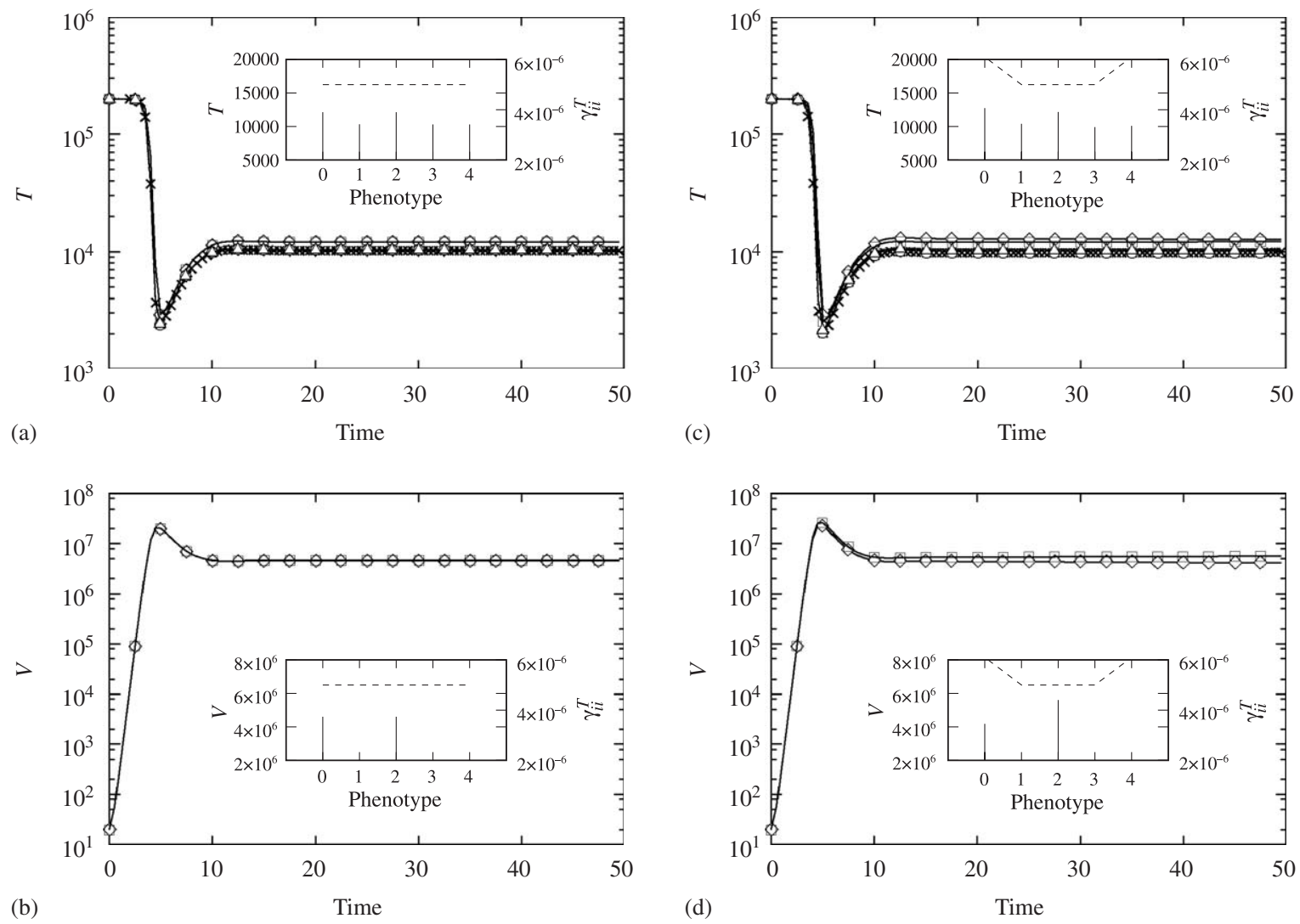

Fig. 6. Time evolution of $\mathrm{T}((\mathrm{a})$ and (c)) and $\mathrm{V}((\mathrm{b})$ and (d)) populations after an inoculum with two strains (phenotypes 0 and 2$), \mu=0$, $A=5 \times 10^{-6}, \varepsilon_{A}=10, N=5$. (a) and (b): $B=0$; (c) and (d): $B^{(T)}=3 \times 10^{-6}, \varepsilon_{B}^{(T)}=10$. In the inset the asymptotic distribution of T and $\mathrm{V}$ populations and the shape of the diagonal of $\gamma^{(T)}$ are shown.

\subsection{Superinfection dynamics}

Results of short time and long term viral coevolution after superinfection are reported in Figs. 7 and 8 , respectively. In both figures the first viral inoculum occurs at time $t=0$ and the second infection at time $t=20$, when the immune response to the first inoculum has completed and the virus has established a chronic infection. After the second inoculum and a short transient, a slow mounting of the second viral infection occurs. This low dynamics continues on a scale of several months (Fig. 8) and eventually reaches the same level of the other quasispecies. This behavior represents another example of a slow relaxation to a fixed-point equilibrium. With the progression of the disease, when the immune system is compromised (low number of $\mathrm{T}$ cells, i.e., low $\lambda$ ), a second inoculum strain requires a very long time to reach the same abundances of the first strain (Fig. 8b).

Experimental evidences [13] show that the probability of observing new fitter recombinant strains increases with the number of already coevolving strains. This effect has not been yet incorporated into our model.

\subsection{Coevolution and speciation}

Finally, we have studied virus quasispecies formation in more detail. Fig. 9a shows the initial inoculum at phenotype 15 in a space of 25 , followed by a delayed inoculum at phenotype 5 at time $t=1$. The non-uniform interaction strength would favor the central phenotypes. However, since in this simulation the immune system does not discriminate among similar phenotypes (modeled by $\varepsilon_{A}^{(T)}=10$ ), there is an induced competition among neighboring strains. This competition induces a separation of the original quasispecies into two 

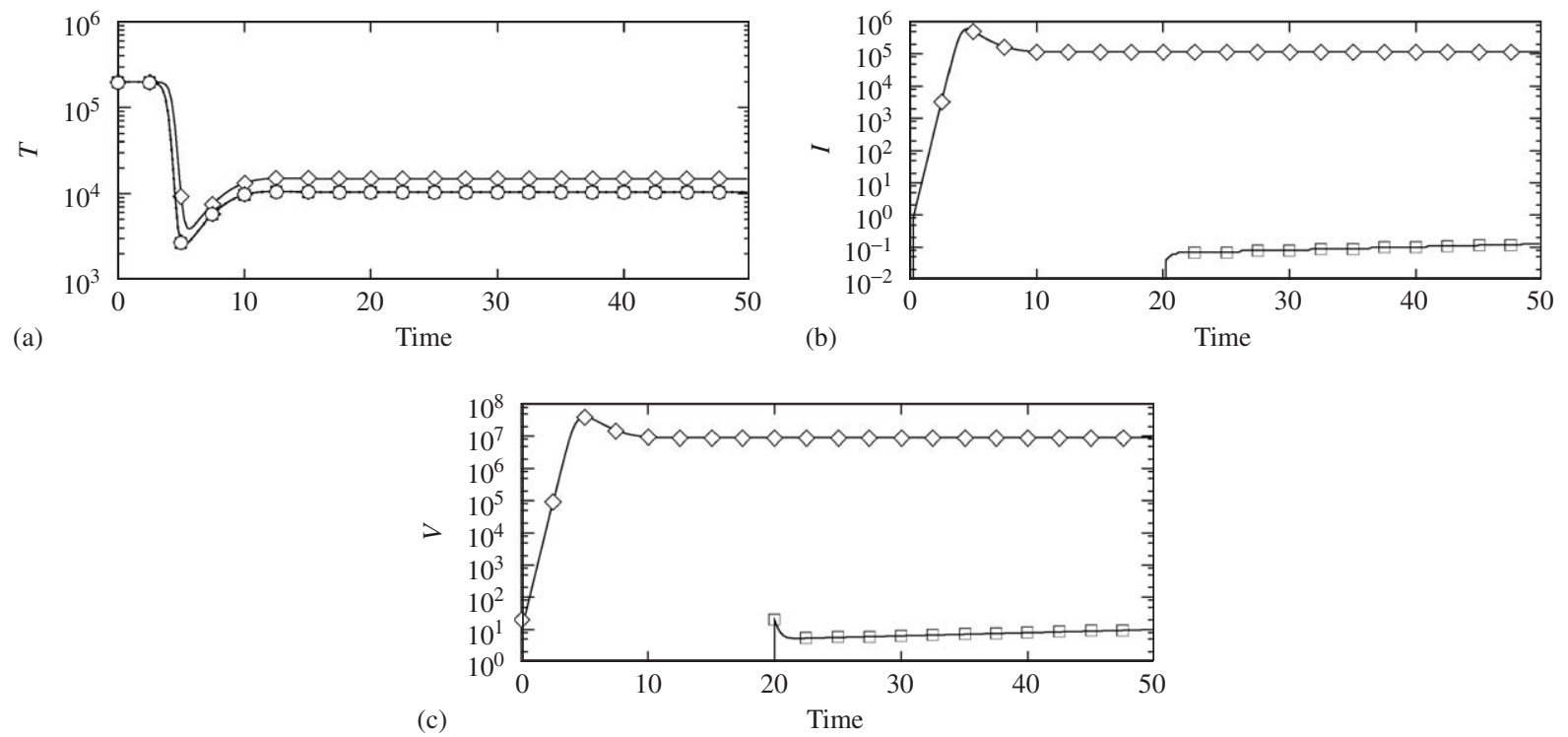

Fig. 7. Short time behavior of (a) uninfected T cells, (b) infected T cells and (c) viruses under superinfection. Parameters as in Fig. 6a, b.
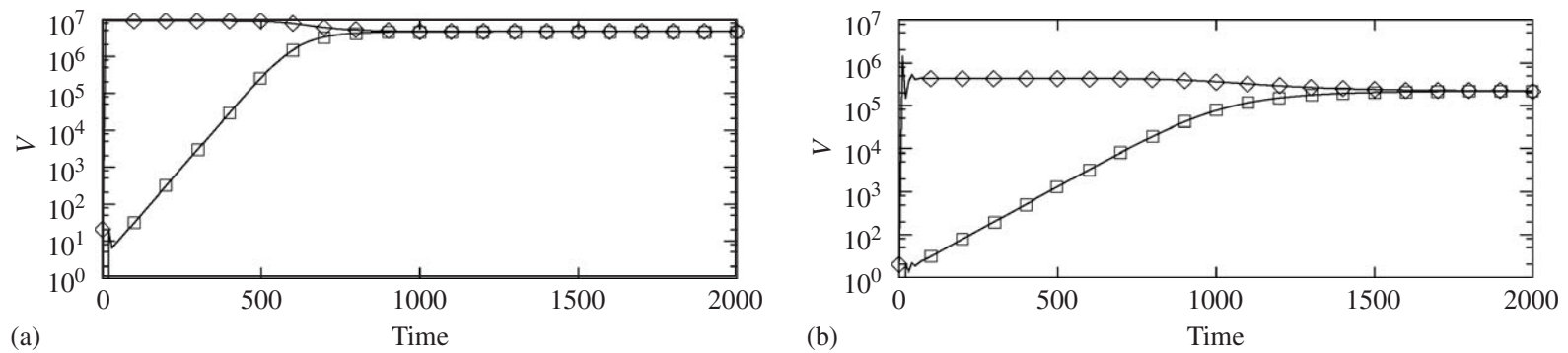

Fig. 8. Long time behavior of virus abundances after superinfection at time $t=20$. (a) healthy immune system $\left(\lambda=10^{5}\right)$; $(\mathrm{b})$ weak immune system $\left(\lambda=10^{4}\right)$. The other parameters used have the same values of Fig. $6 \mathrm{a}$, b. Diamonds represent the first inoculum and squares the second inoculum.

clusters (quasispeciation), Fig. 9b. However, as shown in Figs. 9c, d the immune system response continues to change in time, resulting in a complex coevolution with viral populations. Fig. 10 shows the typical irregular evolution of a speciation dynamics.

\section{Discussion}

We presented a model of the within-patience persistence of HIV quasispecies. We have first extended Perelson's standard model [27] to incorporate B cell response. The B cells, once activated by specific T cells, can only act as a predator, and not being directly targeted as a prey by HIV. We found that this role represents a non-negligible contribution to the immune response in all cases where a virus or bacterium is targeting (and being targeted by) $\mathrm{T}$ cells. Interestingly, $\mathrm{T}$ cells have a role in both innate and adaptive immune responses while B cells only in the adaptive system. It is known that the innate immune response is present also in insects while the adaptive is more recent, being present only in vertebrate. Thus, B cells may have appeared also to fight back viruses targeting specifically at $\mathrm{T}$ cells.

Recent works have shown that HIV quasispecies may compete [11] and that persistence of the initial or ancestor quasispecies is a good indicator for disease progression [12]. 


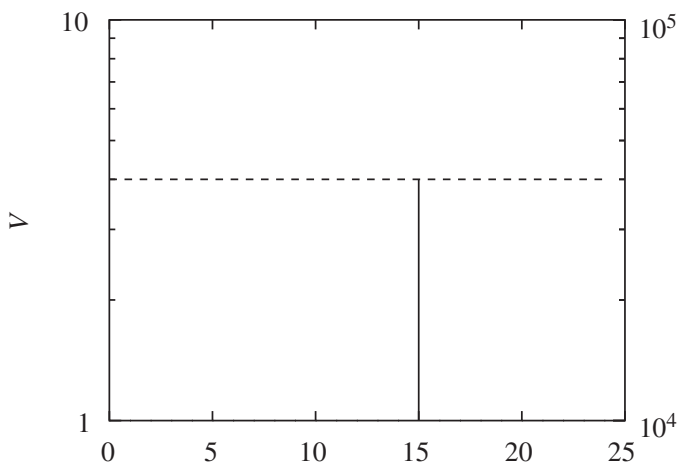

(a)

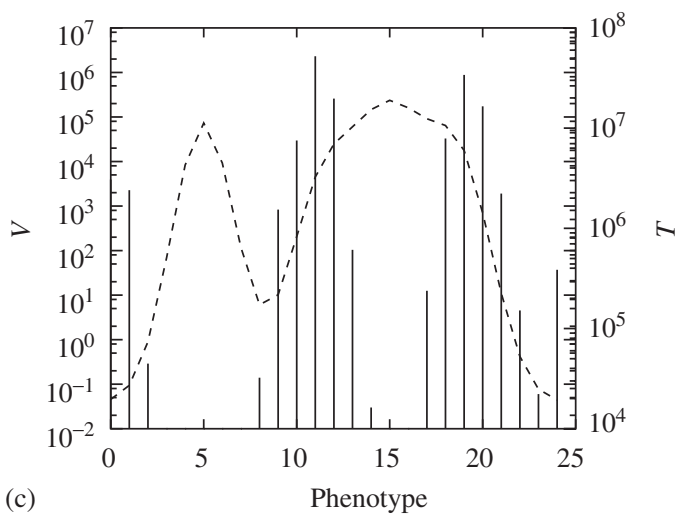

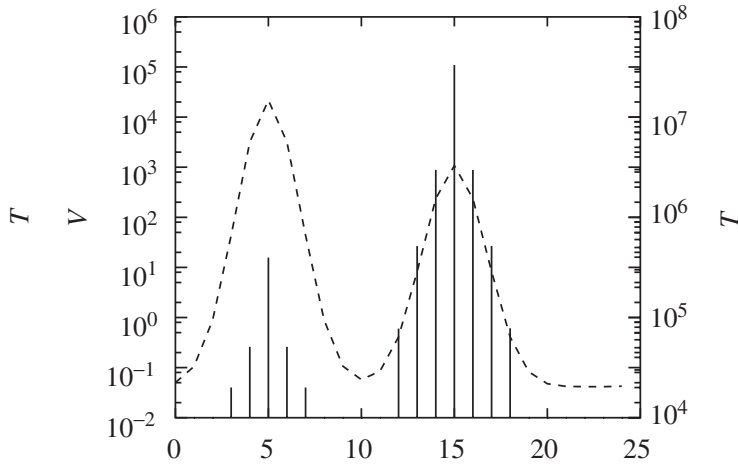

(b)

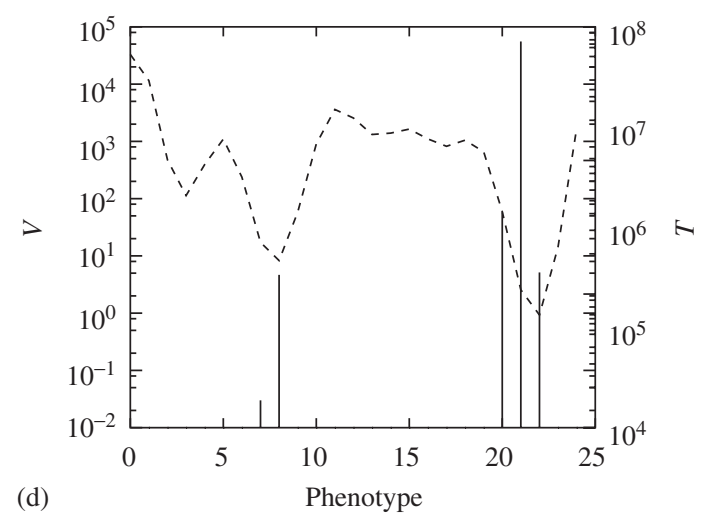

Fig. 9. Speciation of virus quasispecies and uninfected T cells dynamics after competitive superinfection at four different times: (a) $t=0$, (b) $t=4.5$, (c) $t=5.25$ and (d) $t=5.75$. Virus strain 15 is present at time $t=0$, while strain 5 is inoculated at time $t=1$. Mutation rate $\mu=10^{-4}$ and non-uniform interaction strength as in Fig. $6 \mathrm{c}, \mathrm{d}$. The dashed line represents the abundances of $\mathrm{T}$ cells targeting each viral phenotype.

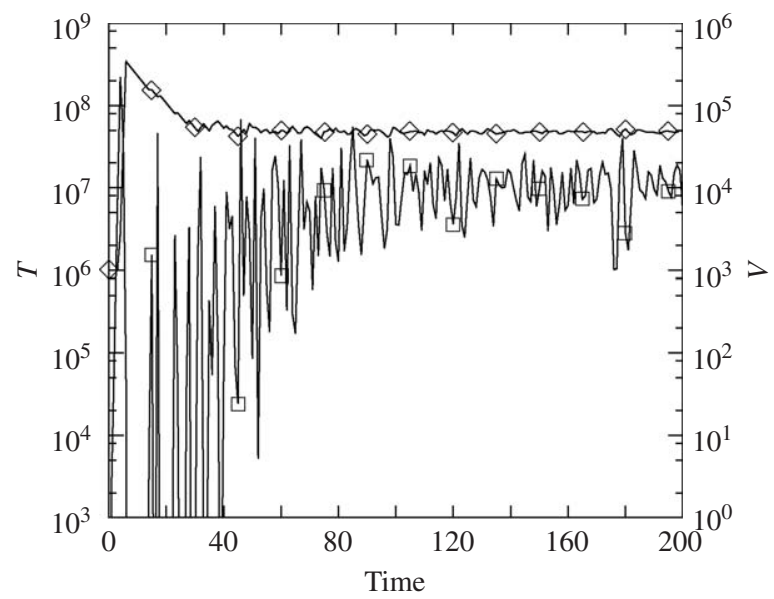

Fig. 10. Temporal evolution of speciation of virus quasispecies and uninfected $\mathrm{T}$ cell dynamics after superinfection; diamonds represent uninfected T cells, squares represent viruses. Virus strain with phenotype 15 is present at time $t=0$ and the strain with phenotype 5 is inoculated at time $t=1$. We set $A^{(T)}=5 \times 10^{-5}, A^{(I, V)}=5 \times 10^{-6}, \varepsilon_{A}=10, B^{(T)}=3 \times 10^{-6}, \varepsilon_{B}^{(T)}=10$, with mutation rate $\mu=10^{-4}$. 
Our model shows that the time evolution of the competition between quasispecies is slow and has time scales of several months. This provides a hint of why standard viral dynamics models, which ignore multiple infections, are effective in describing viral load evolution in HIV-infected individuals.

Burch and Chao [38] have stressed that the evolution of an RNA virus is determined by its mutational neighborhood. As the phenotype divergence among viral strains arises from differences in selection pressure, these differences may lead, for instance, to a higher infection rate. Since the competition is through the immune system response and given that the phase space of antigen recognition is not homogeneously covered [14], the HIV high mutation rate allows the quasispecies to find regions with weak immune response. This competition may lead to speciation of viral strains.

Work in progress considers parametric time dynamics; this approach would allow for instance to model the shift in usage from CCR5 to CXCR4 chemokine receptors, corresponding to a change in viral selection [43].

The introduction or modulation of a quasispecies may be used in therapy against an already present aggressive strain. This would be particularly effective during full AIDS stage when virus burden is particularly high and the conditions for competition are more stringent. It is noteworthy that Schnell and colleagues [39] have constructed a recombinant vesicular stomatitis virus that although unable to infect normal cells, infected and killed cells that were first infected with HIV causing a rapid cytopathic infection. The authors showed that the introduction of this engineered virus can achieve HIV load reduction of $92 \%$ and recovery of host cells to $17 \%$ of their normal levels (see also the mathematical model in Ref. [40]).

In summary, our model represents a general framework to investigate several aspects of the evolution of HIV infections, for example intermittency or switching dominance of strains and the arising of new dominant strains during different phases of therapy; how superinfection will evolve in case of replacement of drugresistant virus with a drug-sensitive virus and acquisition of highly divergent viruses of different strains. It is also useful to investigate whether antiviral treatment may increase susceptibility to superinfection by decreasing antigen load. Different drug treatments can alter the population of quasispecies. Quasispecies may be the key to understanding the emerging infectious diseases and has implications for transmission, public health counselling, treatment and vaccine development.

\section{References}

[1] J. Parkin, B. Cohen, Lancet 357 (2001) 1778.

[2] A.S. Perelson, G. Weisbuch, Rev. Mod. Phys. 576 (1997) 51.

[3] G. Pantaleo, C. Graziosi, A.S. Fauci, N. Engl. J. Med. 328 (1993) 327.

[4] G.H. Kijak, E. Sanders-Buell, N.D. Wolfe, E. Mpoudi-Ngole, B. Kim, B. Brown, M.L. Robb, D.L. Birx, D.S. Burke, J.K. Carr, F.E. McCutchan, AIDS Res. Hum. Retroviruses 20 (2004) 521.

[5] O.O. Yang, E.S. Daar, B.D. Jamieson, A. Balamurugan, D.M. Smith, J.A. Pitt, C.J. Petropoulos, D.D. Richman, S.J. Little, A.J. Leigh-Brown, J. Virol. 79 (2005) 860.

[6] M. Eigen, J. McCaskill, P. Schuster, Adv. Chem. Phys. 75 (1989) 149.

[7] A.U. Neumann, N.P. Lam, H. Dahari, D.R. Gretch, T.E. Wiley, T.J. Layden, A.S. Perelson, Science 282 (1998) 103.

[8] C. Krempl, B. Schultze, H. Laude, G. Herrler, J. Virol. 71 (1997) 3285.

[9] S. Franz, L. Peliti, J. Phys. A 30 (1997) 4481.

[10] M. Eigen, P. Schuster, Naturwissenschaften 64 (1977) 541.

[11] L. Costa, M. Janini, M. Pinto, R. Diaz, Int. Conf. AIDS 13 (2000) MoPpA1005.

[12] G. Bello, C. Casado, V. Sandonis, M. Alonso-Nieto, J.L. Vicario, S. García, V. Hernando, C. Rodríguez, J. del Romero, C. LópezGalíndez, J. Gen. Virol. 86 (2005) 355.

[13] A.E. Jetzt, H. Yu, G.J. Klarmann, Y. Ron, B.D. Preston, J.P. Dougherty, J. Virol. 74 (2000) 1234.

[14] R.J. De Boer, L.A. Segel, A.S. Perelson, J. Theor. Biol. 71 (1992) 295.

[15] D.D. Ho, A.U. Neumann, A.S. Perelson, W. Chen, J.M. Leonard, M. Markowitz, Nature 373 (1995) 123.

[16] J.T. Blackard, D.E. Cohen, K.H. Mayer, Clin. Infect. Dis. 34 (2002) 1108.

[17] A. Ramos, D.J. Hu, L. Nguyen, K.O. Phan, S. Vanichseni, N. Promadej, K. Choopanya, M. Callahan, N.L. Young, J. McNicholl, T.D. Mastro, T.M. Folks, S. Subbarao, J. Virol. 76 (2002) 7444.

[18] J.A. Levy, Lancet 361 (2003) 98.

[19] P.T. Haaft, E.J. Verschoor, B. Verstrepen, H. Niphuis, R. Dubbes, W. Koornstra, W. Bogers, B. Rosenwirth, J.L. Heeney, J. Gen. Virol. 85 (2004) 3735.

[20] M. Altfeld, T.M. Allen, X.G. Yu, M.N. Johnston, D. Agrawal, B.T. Korber, D.C. Montefiori, D.H. O'Connor, B.T. Davis, P.K. Lee, E. Maier, J. Harlow, P.J.R. Goulder, C. Brander, E.S. Rosenberg, B.D. Walker, Nature 420 (2002) 434. 
[21] M. Gerhardt, D. Mloka, S. Tovanabutra, E. Sanders-Buell, O. Hoffmann, L. Maboko, D. Mmbando, D.L. Birx, F.E. McCutchan, M. Hoelscher, J. Virol. 79 (2005) 8249.

[22] P.M. de A. Zanotto, E.A. Gould, G.F. Gao, H.P. Harvey, E.C. Holmes, Proc. Natl. Acad. Sci. USA 93 (1996) 548.

[23] D.L. Chao, M.P. Davenport, S. Forrest, A.S. Perelson, J. Theor. Biol. 228 (2004) 227.

[24] F. Celada, P.E. Seiden, Eur. J. Immunol. 26 (1996) 1350.

[25] R.J. De Boer, A.S. Perelson, J. Theor. Biol. 175 (1995) 567.

[26] D. Wodarzl, M.A. Nowak, BioEssays 24 (2002) 1178.

[27] A.S. Perelson, A.U. Neumann, M. Markowitz, J.M. Leonard, D.D. Ho, Science 271 (1996) 1582.

[28] F.W. Wiegel, A.S. Perelson, Immunol. Cell Biol. 82 (2004) 127.

[29] X. Wei, S.K. Ghosh, M.E. Taylor, V.A. Johnson, E.A. Emini, P. Deutsch, J.D. Lifson, S. Bonhoeffer, M.A. Nowak, B.H. Hahn, M.S. Saag, G.M. Shaw, Nature 373 (1995) 117.

[30] M. Dixit, A.S. Perelson, Proc. Natl. Acad. Sci. 102 (2005) 8198.

[31] M.G. Jenkins, A. Rambaut, O.G. Pybus, E.C. Holmes, J. Mol. Evol. 54 (2002) 156.

[32] L.M. Mansky, H.M. Temin, J. Virol. 69 (1995) 5087.

[33] P. Lio, N. Goldman, Trends Microbiol. 12 (2004) 106.

[34] F. Bagnoli, M. Bezzi, Phys. Rev. Lett. 79 (1997) 3302.

[35] M.J. Miller, O. Safrina, I. Parker, M.D. Cahalan, J. Cell Biol. 167 (2004) i1.

[36] C.K. Biebricher, M. Eigen, Virus Res. 107 (2005) 117.

[37] A.P. Galvani, M. Slatkin, Proc. Natl. Acad. Sci. USA 100 (2003) 15276.

[38] C.L. Burch, L. Chao, Nature 406 (2000) 625.

[39] M.J. Schnell, E. Johnson, L. Buonocore, J.K. Rose, Cell 90 (1997) 849.

[40] T. Revilla, G. Garcýa-Ramos, Math. Biosci. 185 (2003) 191.

[41] J.R. Gog, B.T. Grenfell, Proc. Natl. Acad. Sci. 99 (2002) 17209.

[42] J. Lin, V. Andreasen, R. Casagrandi, S.A. Levin, J. Theor. Biol. 222 (2002) 437.

[43] R. Shankarappa, et al., J. Virol. 73 (1999) 10489. 